\title{
Cardiovascular Autonomic Dysfunction in Type-2 Diabetes patients with Microalbuminuria in a Tertiary Level Hospital
}

\author{
MD. RUBEL MIAH, ${ }^{1}$ MD. SAIDUR RAHMAN, ${ }^{2}$ SARMISTHA BISWAS, ${ }^{3}$ GOBINDA CHANDRA BANIK, ${ }^{3}$ \\ MD. FAIZUL ISLAM CHOWDHURY4
}

\begin{abstract}
Background: Cardiovascular autonomic dysfunction is a serious complication of diabetes mellitus (DM) and is independently associated with microalbuminuria in patients with type-2 DM.This study was conducted to determine the prevalence of cardiovascular autonomic dysfunction in type-2 diabetes patients with microalbuminuria.

Materials \& Methods: This descriptive type of observational study was done in the department of Medicine, Dhaka Medical College Hospital from August 2015 to January 2016.

Results: A total of 50 patients was included in the study with male predominance (M:F ratio 2. 22:I). Peak age was between 40-60 years and majority of patients (32\%) was between $4 I-50$ years and mean duration of DM was more than 12 years. Out of 50 patients, 19 (38\%) patients had mild microalbuminuria; 24 (48\%) patients had moderate microalbuminuria and 7 (14\%) patients had severe microalbuminuria. Common symptoms were lethargy, anorexia, nausea, vomiting, dizziness and bladder symptoms. Cardiac autonomic neuropathy (CAN) was found in $80 \%$ cases. Parasympathetic dysfunction was observed in 40 (80\%) cases and sympathetic dysfunction was observed in 43 (86\%) cases.

Conclusion: As significant number of DM patients (80\%) develop CAN, so cardiovascular autonomic function tests should be monitored to address major potential cardiovascular complications even in asymptomatic patients, especially among those with microalbuminuria.
\end{abstract}

Key words: cardiovascular autonomic dysfunction (CAD); type-2 DM; microalbuminuria.

DOI: http://dx.doi.org//0.3329/jom.v19i2.37229

\section{Introduction:}

The prevalence of cardiovascular autonomic dysfunction in diabetes is not precisely known; ${ }^{1}$ Abnormal cardiovascular function tests suggest poor prognosis and increased incidence of silent myocardial infarction, cardiac arrest, sudden death, and inadequate response to stressful events, e. g., anaesthesia and surgery. ${ }^{2,3}$ There are very few reports about the prevalence of cardiac autonomic neuropathy (CAN) along with correlation with other diabetic complications. To highlight the magnitude of the

1. Assistant Registrar, Department of Medicine, Dhaka Medical College Hospital

2. Indoor Medical Officer, Department of Medicine, Dhaka Medical College Hospital

3. Associate Professor, Department of Medicine, Dhaka Medical College Hospital

4. Professor, Department of Medicine, Dhaka Medical College Hospital

Corresponding author: Dr. Md. Rubel Miah, Assistant Registrar, Department of Medicine, Dhaka Medical College Hospital. Email: rubelmd22@gmail.com.

Received: 21 January 2018;

Accepted: 19, April 2018 problem in Bangladeshi population, the present study was planned.

Microalbuminuria is recognized as the earliest marker of renal disease and is also strongly associated with increased risk of cardiovascular complications, including atherosclerotic coronary artery disease, stroke, peripheral vascular disease, and cardiovascular mortality. This association is independent of other known cardiovascular risk factors such as hypertension, dyslipidaemia, obesity, smoking, and impaired renal function. Cardiovascular autonomic dysfunction (CAD) could potentially constitute another such mechanism. Indeed, few studies have previously shown that $\mathrm{CAD}$ is associated with microalbuminuria, especially in individuals with impaired glucose metabolism and diabetes mellitus. ${ }^{4,5}$

Several studies have suggested that impaired cardiovascular autonomic function and increased urinary albumin excretion are related in patients with diabetes. Most of these studies have been done in type 1 diabetes. Similar studies in type 2 diabetes were relatively small. ${ }^{6}$ 
For that reason, we planned to investigate the pattern of autonomic dysfunction and prevalence in patients with microalbuminuria and made an attempt to evaluate if there is an independent relation between increased urinary albumin excretion and subclinical autonomic neuropathy.

\section{Materials \& Methods:}

This observational study was done in the department of Medicine, Dhaka Medical College Hospital, Dhaka from August 2015 to January 2016. Hospitalised adult patients with diagnosis of type-2 DM were primarily enrolled for the study. Those with significant microalbuminuria were finally included for analysis. Patients with uncontrolled hypertension, overt albuminuria, cardiac failure, preexisting macrovascular condition, urinary tract infection (all of which could have transient proteinuria), using ACE inhibitors and/ or drugs known to influence autonomic nerve function, pregnant females were excluded from the study. A first morning mid stream urine sample was collected for routine and microscopic examination. Patients with urine positive for protein were excluded from the study and those negative for protein were tested for microalbuminuria with timed urinary collection (24 hrs) by ELISA method. Microalbuminuria defined as AER (albumin excretion rate) $30-300 \mathrm{mg} / 24 \mathrm{hr}$; graded as mild ( $20-50 \mathrm{mg} / 24 \mathrm{hr}$ ), moderate (50-100 mg/24 hr) or severe (100-300 mg/24 hr) depending on the color change in the strip. It was performed by bed side dipstick test.

Cardiovascular autonomic function tests (CAFT) which includes tests for heart rate variability and responses to the certain maneuver were conducted. An electrocardiograph was obtained from bipolar chest leads, R-R intervals were obtained by using a QRS detector with an accuracy of $1 \mathrm{~ms}$. The following measures of cardiovascular autonomic function were used:

- Heart-Rate variation to valsalva maneuver: The subject was seated comfortably and was asked to blow into a mouthpiece which was connected to a mercury sphygmomanometer, holding it at a pressure of $40 \mathrm{~mm}$ $\mathrm{Hg}$ for 15 seconds, while a continuous ECG in lead II was being recorded. The ECG was continued to be recorded for 30 seconds after the release of the pressure at the end of 15 seconds. The heart rate changes which were induced by the Valsalva maneouver were expressed as the ratio of the maximal tachycardia during the maneouver to the maximal bradycardia after the maneouver. This ratio was defined as the Valsalva ratio and was calculated as the ratio of the maximum R-R interval after the maneouver to the minimum R-R interval during the maneouver. Valsalva Ratio $(\mathrm{VR})=$ Maximal tachycardia/maximal bradycardia $=$ Maximum $\mathrm{R}-\mathrm{R}$ interval/minimum R-R interval.

- Deep Breathing test: After 10 min of rest in a supine position, the deep-breathing test was performed. Subject was asked to breathe quietly and deeply at the rate of 6 breaths per minute ( $5 \mathrm{~s}$ in, $5 \mathrm{~s}$ out). A continuous ECG was recorded for six cycles. The maximum and minimum $\mathrm{R}-\mathrm{R}$ intervals in lead II were measured during each breathing cycle and were converted to beats per minute. The result was then expressed as the mean of the difference between the maximum and the minimum heart rate for six measured cycles in beats/minute. Deep Breathing Difference $(D B D)=$ Mean of heart rate differences in 6 breath cycles.

- Heart Rate Response to Standing (Postural tachycardia index): The subjects were asked to lie on the examination table quietly while the heart rate was being recorded on the ECG. They were then asked to stand-up unaided and the ECG was recorded for 1 minute. The shortest R-R interval at or around the 15th beat and the longest R-R interval at or around the 30th beat were measured. The result was expressed as PTI = Longest R-R interval at the 30th beat/shortest R-R interval at the 15 th beat.

- Blood pressure response to standing (Orthostatic test): The subject was asked to rest in a supine position. The resting $\mathrm{BP}$ was recorded by using a sphygmomanometer. The subject was then asked to stand unaided and to remain standing unsupported for 3 minutes. The BP was recorded at 30 seconds and 3 minutes after standing up. The difference between the resting and the standing BP levels was calculated. The fall in systolic BP at 30 seconds on standing was noted.

- Blood pressure response to sustained handgrip: The patient was asked to compress the inflated blood pressure cuff to the maximum possible extent with one hand and the reading on the manometer was noted. The patient was asked to maintain the pressure of the cuff in such a way as to keep the manometer reading at $30 \%$ of the maximum force for a period of 3 minutes. The maximum reading of the diastolic blood pressure was recorded during the procedure. Then, the rise in the diastolic blood pressure was calculated by subtracting the resting diastolic blood pressure from this value. 
Cardiovascular autonomic function tests (CAFT): Cardiac autonomic neuropathy is detected by various cardiac autonomic function tests which include ${ }^{6}$ :

\begin{tabular}{|c|c|c|c|}
\hline Autonomic test & $\begin{array}{c}\text { Normal } \\
\text { (Score 0) }\end{array}$ & $\begin{array}{l}\text { Borderline } \\
\text { (Score 1) }\end{array}$ & $\begin{array}{l}\text { Abnormal } \\
\text { (Score 2) }\end{array}$ \\
\hline $\begin{array}{l}\text { Heart rate response to Valsalva manoeuvre }(15 \mathrm{secs}) \text { : ratio of } \\
\text { longest to shortest R-R interval }\end{array}$ & $>1.21$ & $0-1.20-1.11$ & $<1.10$ \\
\hline $\begin{array}{l}\text { Heart rate response to deep breathing ( } 6 \text { breaths over } 1 \mathrm{~min}) \text { : } \\
\text { maximum-minimum heart rate }\end{array}$ & $>15$ beat $/$ min & $11-14$ beat $/ \mathrm{min}$ & $<10$ beat $/$ min \\
\hline $\begin{array}{l}\text { Heart rate response to standing after lying: ratio of R-R } \\
\text { interval of } 30 \text { th to } 15 \text { th beats }\end{array}$ & $>1.04$ & $1.01-1.03$ & $<1.00$ \\
\hline Blood pressure response to standing: Fall in systolic BP (mm Hg) & $<10 \mathrm{~mm} \mathrm{Hg}$ & $11-29 \mathrm{~mm} \mathrm{Hg}$ & $>30 \mathrm{~mm} \mathrm{Hg}$ \\
\hline $\begin{array}{l}\text { Blood pressure response to sustained hand grip (rise in diastolic } \\
\text { BP): highest diastolic BP during hand grip - mean of } 3 \text { diastolic BP } \\
\text { before hand grip began ( } \mathrm{mm} \mathrm{Hg} \text { ) }\end{array}$ & $>16 \mathrm{~mm} \mathrm{Hg}$ & $11-15 \mathrm{~mm} \mathrm{Hg}$ & $<10 \mathrm{~mm} \mathrm{Hg}$ \\
\hline
\end{tabular}

Results:

A total of 50 patients were taken in the study; male were 34 and female were 16. Majority of the patients was between 41-50 years and mean duration of DM was more than 12 years [table 1]. Common symptoms were lethargy, anorexia, nausea, vomiting, dizziness and bladder symptoms. CAN (total number of patients having abnormal cardiovascular reflexes including one, two or three abnormal reflexes) was found in $80 \%$ cases; Parasympathetic dysfunction was observed in $40(80 \%)$ cases and sympathetic dysfunction was observed in $43(86 \%)$ cases.

Table 1

Base line characteristics of study population $(n=50)$.

\begin{tabular}{lc}
\hline Total number of patients & 50 \\
Peak age (years) & $40-60$ \\
Male:Female & $2.22: 1$ \\
Insulin:Oral medication & $1.63: 1$ \\
Mean duration of DM (years) & $>12$ \\
\hline
\end{tabular}

Regarding the heart rate response to Valsalva maneuver, abnormal response was observed in 19 subject; majority were male 8 (23.53). In case of heart rate response to Deep Breathing, abnormal heart rate was found in 37 patients. In this series, female subjects 8 (50.0) were affected more than male 14 (41.17). No patients were detected with normal heart rate response during Deep Breathing. Heart rate response to standing showed that 23 patients had abnormal heart rate with female 6 (37.5) predominance. Borderline heart rate was found in 20 subjects. So, overall abnormal heart rate was found in 19, 37, 23 number of cases respectively [table II].

Table-II

Heart rate (HR) response in the study group $(n=50)$

\begin{tabular}{lcc}
\hline Variable & Frequency & Total \\
& $M(n=34)$ & $F(n=16)$ \\
\hline
\end{tabular}

Heart rate response to Valsalva Maneuver (R-R interval)

$\begin{array}{lll}<0.5 & 7(20.58) & 3(18.75) \\ 0.6-10\end{array}$

$\begin{array}{lll}0.6-1.10 & 8(23.53) & 1(6.25) 9\end{array}$

$\begin{array}{lll}1.11-1.15 & 12(35.29) & 5(31.25)\end{array}$

$\begin{array}{lll}1.16-1.20 & 5(14.70) & 6(37.5) 11\end{array}$

$\begin{array}{lll}>1.21 & 2(5.88) & 1(6.25) 3\end{array}$

Heart rate response to Deep Breathing

$\begin{array}{lll}1-5 & 9(26.47) & 6(37.5) \\ 6-15\end{array}$

$6-10 \quad 14(41.17) \quad 8(50.0) 22$

$11-14 \quad 11(32.85) \quad 2(12.5) 13$

$15-20$

0

00

Heart rate response to standing

\begin{tabular}{lcl}
$<0.5$ & $9(26.47)$ & $6(37.5) 15$ \\
$0.6-1.0$ & $5(14.70)$ & $3(18.758$ \\
$1.01-1.03$ & $14(41.17)$ & $6(37.5) 20$ \\
$1.04-1.09$ & $4(11.76)$ & $1(6.25) 5$ \\
\hline
\end{tabular}

Severity of microalbuminuria is a prime risk factor for autonomic neuropathy. Finding showed that severe grade 
of microalbuminuria patients is associated with abnormal to borderline dysfunction. In case of heart rate response to valsalva maneuver, 6 (85.71) patients with severe microalbuminuria had abnormal heart rate and 15 (78.94) patients with mild microalbuminuria had borderline heart rate response. All cases of severe microalbuminuric patients had abnormal heart rate response to Deep Breathing, followed by 21 (87.5) patients with moderate microalbuminuria. Heart rate response to standing showed that 14 (73.68) patients with mild microalbuminuria had borderline heart rate response, 18 (75) patients with moderate microalbuminuria and 5 (71.42) patients with severe microalbuminuria had abnormal heart rate response [table III].

Table-III

Association of heart rate (HR) response in different grade of microalbuminuria $(n=50)$

\begin{tabular}{|c|c|c|c|c|}
\hline \multirow[t]{2}{*}{ Variable } & \multicolumn{3}{|c|}{ Grade of microalbuminuria } & Total \\
\hline & $\begin{array}{c}\text { Mild } \\
(n=19)\end{array}$ & $\begin{array}{c}\text { Moderate } \\
(n=24)\end{array}$ & $\begin{array}{c}\text { Severe } \\
(n=7)\end{array}$ & \\
\hline
\end{tabular}

Heart rate response to Valsalva Maneuver (score)

$\begin{array}{lcccc}0 \text { (normal) } & 3(15.78) & 0 & 0 & 3 \\ 1 \text { (borderline) } & 15(78.94) & 12(50.0) & 1(14.28) & 28 \\ 2 \text { (abnormal) } & 1(5.26) & 12(50.0) & 6(85.71) & 19\end{array}$

Heart rate response to Deep Breathing (score)

$\begin{array}{lcccc}0 \text { (normal) } & 0 & 0 & 0 & 0 \\ 1 \text { (borderline) } & 10(52.63) & 3(12.5) & 0 & 13 \\ 2 \text { (abnormal) } & 9(47.36) & 21(87.5) & 7(100.0) & 37\end{array}$

Heart rate response to standing (score)

\begin{tabular}{lcccc}
0 (normal) & $5(26.31)$ & $2(8.3)$ & 0 & 7 \\
1 (borderline) & $14(73.68)$ & $4(16.6)$ & $2(28.57)$ & 20 \\
2 (abnormal) & 0 & $18(75)$ & $5(71.42)$ & 23 \\
\hline
\end{tabular}

Cardiovascular autonomic dysfunction is associated with microalbuminuria in patients with type 2 diabetes mellitus. In this figure mean heart rate score was higher (7.56) in type 2 DM with severe microalbuminuria patients. A positive correlation was observed between the parasympathetic function and the severity of microalbuminuria. Mean score was 6.23 in type $2 \mathrm{DM}$ with mild microalbuminuria, 7. 1 was in type $2 \mathrm{DM}$ with moderate microalbuminuria and 7.566 was in type $2 \mathrm{DM}$ with severe microalbuminuria patients [figure 1]

$\mathrm{BP}$ response to standing demonstrated that total 3 patients had normal systolic BP. In this series maximum were male subjects 2 (5.88). Borderline BP response was detected in 31 cases. Among them 5 (31.25) were female. Abnormal BP response was found in 16 subjects of whom 11 (32.35) were male. BP response to sustained handgrip represented that

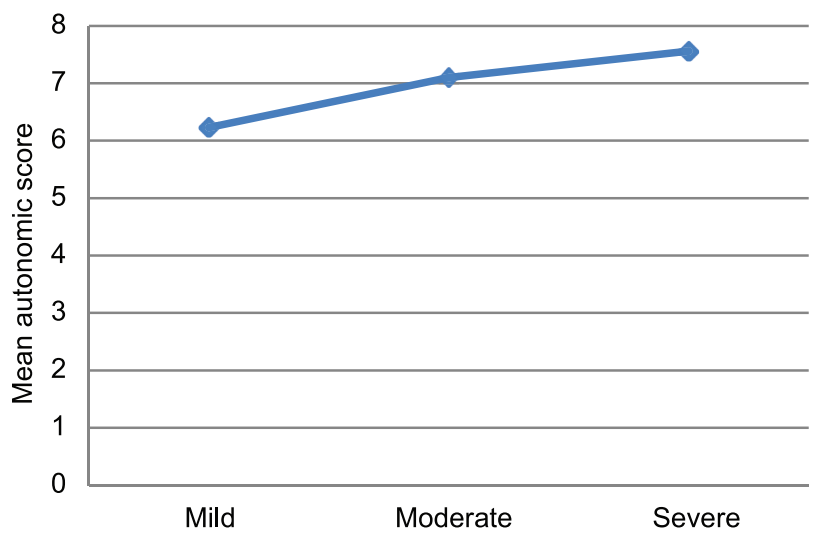

Figure 1: Heart rate response (parasympathetic function) in DM case with different grade of microalbuminuria $(n=50)$.

29 patients had abnormal BP response with female predominance. Borderline BP response was found in 17 subjects and among them 12 (35.29) were male and 5 (31.25) were female [table IV].

\section{Table-IV}

Blood pressure response in the study group $(n=50)$.

\begin{tabular}{|c|c|c|c|}
\hline \multirow[t]{2}{*}{ Variable } & \multicolumn{2}{|c|}{ Frequency } & \multirow[t]{2}{*}{ Total } \\
\hline & $M(n=34)$ & $F(n=16)$ & \\
\hline \multicolumn{4}{|c|}{ BP response to standing: fall in systolic $\mathrm{BP}(\mathrm{mm}$ of $\mathrm{Hg})$} \\
\hline$<5$ & $1(2.94)$ & 0 & 1 \\
\hline $6-10$ & $2(5.88)$ & 0 & 2 \\
\hline $11-20$ & $9(26.47)$ & $3(18.75)$ & 12 \\
\hline $21-29$ & $11(32.35)$ & $8(50.0)$ & 19 \\
\hline$>30$ & $11(32.35)$ & $5(31.25)$ & 16 \\
\hline \multicolumn{4}{|c|}{$\begin{array}{l}\mathrm{BP} \text { response to sustained handgrip: rise in diastolic } \mathrm{BP}(\mathrm{mn} \\
\text { of } \mathrm{Hg})\end{array}$} \\
\hline$<5$ & $2(5.88)$ & $3(18.75)$ & 8 \\
\hline $6-10$ & $14(41.17)$ & $7(43.75)$ & 21 \\
\hline $11-15$ & $12(35.29)$ & $5(31.25)$ & 17 \\
\hline $16-20$ & $2(5.88)$ & $1(6.25)$ & 3 \\
\hline$>20$ & $1(2.94)$ & 0 & 1 \\
\hline
\end{tabular}

Grading of microalbuminuria is a predictor for autonomic neuropathy ,mainly in abnormality of diastolic pressure . Finding showed that severe grade of microalbuminuric patients is associated with abnormal to borderline dysfunction. In BP response to standing, 5 (71.42) patients with severe microalbuminuria had abnormal BP response and 16 (84.21) patients with mild microalbuminuria had borderline response. In case of BP response to sustained handgrip, almost all cases with severe microalbuminuria and 20 (83.3) patients with moderate microalbuminuria had abnormal BP response [table V]. 
Table-V

Association of blood pressure response in different grade of microalbuminuria $(n=50)$.

\begin{tabular}{|c|c|c|c|c|}
\hline \multirow[t]{2}{*}{ Variable } & \multicolumn{3}{|c|}{ Grade of microalbuminuria } & Total \\
\hline & $\begin{array}{l}\text { Mild } \\
(n=19)\end{array}$ & $\begin{array}{c}\text { Moderate } \\
\qquad(\mathrm{n}=24)\end{array}$ & $\begin{array}{c}\text { Severe } \\
(n=7)\end{array}$ & \\
\hline
\end{tabular}

$\mathrm{BP}$ response to standing (score)

$\begin{array}{lcccc}0 \text { (normal) } & 2(10.52) & 1(4.16) & 0 & 3 \\ 1 \text { (borderline) } & 16(84.21) & 13(54.16) & 2(28.57) & 31 \\ 2 \text { (abnormal) } & 1(5.26) & 10(41.66) & 5(71.42) & 16\end{array}$

BP response to sustained handgrip (score)

\begin{tabular}{lcccc}
0 (normal) & $3(15.78)$ & 0 & $1(14.28)$ & 4 \\
1 (borderline) & $13(68.42)$ & $4(16.6)$ & 0 & 17 \\
2 (abnormal) & $3(15.78)$ & $20(83.3)$ & $6(85.71)$ & 29 \\
\hline
\end{tabular}

Present study demonstrated that cardiovascular autonomic function (sympathetic function) is related to the presence of microalbuminuria. In this figure mean BP score was 6. 58 in type $2 \mathrm{DM}$ patients with mild microalbuminuria, 7.42 was in type $2 \mathrm{DM}$ patients with moderate microalbuminuria and 8.36 was in type $2 \mathrm{DM}$ patients with severe microalbuminuria. Microalbuminuria is a strong and independent indicator of increased cardiovascular risk among individuals with diabetes [figure 2].

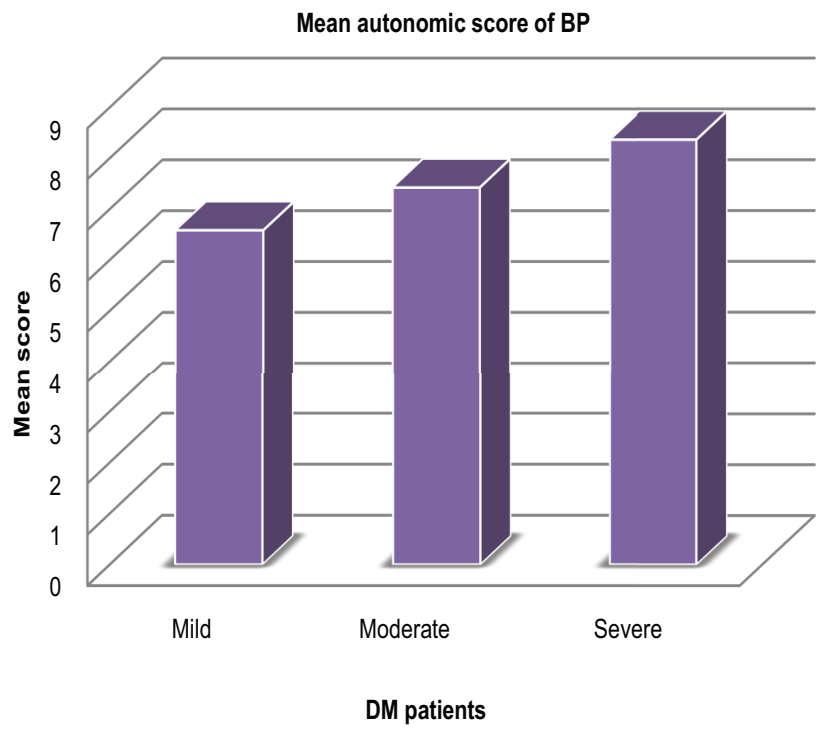

Figure 2: Blood pressure (BP) response (Sympathetic Function) in DM case with different grade of microalbuminuria $(n=50)$.

\section{Discussion:}

In this observational study, we tried to evaluate the prevalence of cardiovascular autonomic dysfunction in type2 diabetes patients with microalbuminuria in a tertiary level hospital of Bangladesh.

Peak age was between $40-60$ years, maximum patients (32\%) were between 41-50 (years) age groups, male-female ratio was 2.22:1. Among the risk factors hypertension was the most common (76\%), next common risk factors were smoking (66\%); obesity (54\%); dyslipidaemia (32\%). Mean duration of DM was more than 12 years. About $62 \%$ patients had history of taking injectable (insulin) medication for diabetes, $38 \%$ received the oral medication. Present study showed that, $40(80 \%)$ patients had parasympathetic dysfunction (at least one of the three tests of parasympathetic function being abnormal) and $43(86 \%)$ patients had sympathetic dysfunction (at least one of the two tests of sympathetic function being abnormal) which is consistent to the study done by Basu AK in which parasympathetic and sympathetic abnormality were $52 \%$ and $20 \%$ respectively. ${ }^{2}$

In this study, common risk factors were HTN, smoking, obesity and dyslipidaemia and it is consistent with the study done by Deepak N Parchwani et al. ${ }^{7}$ in which age, triglycerides, blood pressure and $\mathrm{HbA} 1 \mathrm{c}$ were significantly related to the presence of microalbuminuria.

Present study demonstrated that cardiovascular autonomic dysfunction is related to the presence of microalbuminuria which is consistent with other international studies. ${ }^{4}$

\section{Conclusions:}

Evaluation of cardiovascular reflexes in type 2 DM subjects with paucity of related symptoms constitutes an important feasible and reproducible beside clinical technique and correlates with abnormal albumin excretion. It should be included as a routine in work-up of patients with type 2 DM as it often uncovers autonomic neuropathy even in asymptomatic state.

\section{Conflict of interest: None}

\section{References:}

1. Sudhanva S. Cardiovascular Autonomic Function in Microalbuminuria. Journal of Clinical and Diagnostic Research. 2011 August, Vol-5 (4): 801-803.

2. Basu AK. A Study on the Prevalence of Cardiac Autonomic Neuropathy in Type-2 Diabetes in Eastern India. JIACM 2010; 11 (3): 190-4. 
3. Maser RE, Pfeifer MA, Dorman JS, Kuller. Diabetic Autonomic Neuropathy and Cardiovascular Risk: Pittsburgh Epidemiology of Diabetes Complications Study III. Arch Intern Med 1990;150:1218-22.

4. Smulders YM, Jager A, Gerritsen J, Dekker JM, Nijpels G, Heine RJ et al. Cardiovascular autonomic function is associated with (micro-)albuminuria in elderly Caucasian subjects with impaired glucose tolerance or type 2 diabetes: the Hoorn Study. Diabetes Care 2000;23:1369-1374.

5. Hanneke JBH, Beijers, Isabel F, Bert B, Dekker JM, Nijpels Get al. Microalbuminuria and Cardiovascular Autonomic Dysfunction Are IndependentlyAssociated With
Cardiovascular Mortality:Evidence for Distinct Pathways. Diabetes Care 2009;32:1698-1703.

6. Gerstein HC; Mann JF; Yi Q; Albuminuria and Risk of Cardiovascular Events, Death, and Heart Failure in Diabetic and Nondiabetic Individuals. JAMA, July 25, 2001-Vol 286, No. 4.

7. Parchwani DN, Dhanani JV, Upadhyah AA, Sharma MH, Shah AM, Maheria PB, et al. Relationship between cardiovascular autonomic (dys)function and microalbuminuria in type 2 diabetes mellitus. Natl J Physiol Pharm Pharmacol 2012; 2:84-92. 